\title{
Exponential stability of the continuous method model for solving general variational inequality problems
}

\author{
Wenjie Xu, Yigui Ou* \\ Department of Mathematics, Hainan University, Haikou 570228 China \\ *Corresponding author, e-mail: ouyigui@126.com
}

Received 31 Jul 2020

Accepted 7 Oct 2020

\begin{abstract}
In Ou and Lin [Int J Comput Math 93(2016):1899-1920], a continuous method model for solving general variational inequality problems with convex constraints was proposed. However, only the convergence property of the proposed model was established, while the stability property was not analyzed. In this paper, we further study the proposed model and establish its exponential stability property under some mild conditions. Furthermore, the stability results on two special cases are also obtained under weaker conditions.
\end{abstract}

KEYWORDS: general variational inequality, continuous method model, exponential stability

MSC2010: 65K05 90C30 49M37

\section{INTRODUCTION}

In this paper, we are concerned with the following general variational inequality problem with convex constraints, denoted by $\operatorname{GVI}(F, G, X)[1]$ : Find a point $x$ such that

$$
G(x) \in X,(y-G(x))^{\mathrm{T}} F(x) \geqslant 0, \quad \forall y \in X,
$$

where $F$ and $G$ are continuous mappings from $\mathbb{R}^{n}$ into $\mathbb{R}^{n}$, and $X$ is a nonempty closed convex subset of $\mathbb{R}^{n}$. For simplicity, we denote the solution set of $\operatorname{GVI}(F, G, X)$ by $X^{*}$.

The general variational inequality problem with convex constraints originates in many applications, see $[2,3]$ for instance. These various applications have provoked many scholars to study efficient methods for solving $\operatorname{GVI}(F, G, X)$ (1). Up to now, these methods are divided into two categories: One is the iterative methods, see $[2,4,5]$ for instance; The other is the the continuous method (or termed 'neurodynamical approach' by Liao et al [6]), which combines the attractive features in both dynamical system and neural network approaches. Here, we only focus on some theoretical analysis of the latter.

Based on the projection operator, Xia and Wang [7] studied the $\operatorname{GVI}(F, G, X)(1)$ and proposed a general projection neural network with its dynamical system (GPNN)

$$
\left\{\begin{array}{l}
\frac{\mathrm{d} x}{\mathrm{~d} t}=\Lambda\left\{P_{X}[G(x)-F(x)]-G(x)\right\} \\
x\left(t_{0}\right)=x_{0}
\end{array}\right.
$$

where $F$ and $G$ are continuously differentiable mappings, $\Lambda$ is a positive diagonal matrix, and $P_{X}: \mathbb{R}^{n} \rightarrow X$ is a projection operator defined as

$$
P_{X}(x)=\underset{y \in X}{\arg \min }\{\|y-x\|\} .
$$

However, the convergence and stability properties of GPNN (2) are established under the assumptions that the Jacobian matrices of $F(x)$ and $G(x)$ exist and $\nabla F(x)+\nabla G(x)$ has an upper bound in $\mathbb{R}^{n}$, and thus the applicable scope of GPNN (2) is reduced. To avoid using any form of matrix information in convergence analysis, Ou and Lin [8] recently investigated the $\operatorname{GVI}(F, G, X)(1)$ and developed a continuous method model with its dynamical system (GPCM)

$$
\left\{\begin{array}{l}
\frac{\mathrm{d} x}{\mathrm{~d} t}=\left\{P_{X}[G(x)-\beta F(x)]-G(x)\right\}, \\
x\left(t_{0}\right)=x_{0}, \quad \forall \beta>0,
\end{array}\right.
$$

where $F$ and $G$ are only assumed to be continuous mappings. It should be mentioned that the convergence property of GPCM (4) is established without requiring the existence of Jacobian matrices of $F(x)$ and $G(x)$, and thus it can be applied to analyze a much wider range of dynamical system.

Although the preliminary numerical simulation results in [8] indicate that the proposed GPCM (4) is more efficient than the GPNN (2) in terms of the CPU time and the final norm of $E(x, \beta)$ (see its definition in the next section), the stability property 
of dynamical system GPCM (4) was not analyzed in [8]. As we know, the dynamical stability property is an important issue in designing a continuous method model [6]. Therefore, it is essential for us to study the stability property of GPCM (4), which is the motivation behind the present study.

Based on the above observations, in this paper, we further study the system GPCM (4) and discuss its stability property. Under some mild conditions, it is shown that the proposed model possesses exponential stability property.

\section{MAIN RESULT}

In this section, we will establish the exponential stability property of GPCM (4). For this purpose, we first introduce several definitions and lemmas, which are useful for our later discussions. For details, see $[8,9]$ for instance.

Definition 1 [8] The mapping $F: \mathbb{R}^{n} \rightarrow \mathbb{R}^{n}$ is said to be:

(1) $G$-monotone at $u^{*} \in \mathbb{R}^{n}$ if $\forall u \in \mathbb{R}^{n}$

$$
\left[F(u)-F\left(u^{*}\right)\right]^{\mathrm{T}}\left[G(u)-G\left(u^{*}\right)\right] \geqslant 0 .
$$

(2) $G$-monotone on $\mathbb{R}^{n}$ if $\forall u, v \in \mathbb{R}^{n}$

$$
[F(u)-F(v)]^{\mathrm{T}}[G(u)-G(v)] \geqslant 0 .
$$

(3) $G$-strongly monotone at $u^{*}$ if there exists a constant $\omega>0$ such that $\forall u \in \mathbb{R}^{n}$

$$
\left[F(u)-F\left(u^{*}\right)\right]^{\mathrm{T}}\left[G(u)-G\left(u^{*}\right)\right] \geqslant \omega\left\|u-u^{*}\right\|^{2} .
$$

Definition 2 [9] The mapping $H: \mathbb{R}^{n} \rightarrow \mathbb{R}^{n}$ is said to be monotone if $\forall x, y \in \mathbb{R}^{n}$

$$
[H(x)-H(y)]^{\mathrm{T}}(x-y) \geqslant 0 .
$$

Especially, $H$ is said to be strongly monotone with modulus $\mu>0$ if $\forall x, y \in \mathbb{R}^{n}$

$$
[H(x)-H(y)]^{\mathrm{T}}(x-y) \geqslant \mu\|x-y\|^{2} .
$$

Lemma 1 [9] Assume that $f: D \subset \mathbb{R}^{n} \rightarrow \mathbb{R}$ is differentiable on an open set $D$, and that $S \subset D$ is a convex subset. Then $f$ is strongly convex on $S$ if and only if its gradient $\nabla f$ is strongly monotone.

Lemma 2 [8] The point $x^{*}$ solves $\operatorname{GVI}(F, G, X)$ (1) if and only if $x^{*}$ is the equilibrium solution of GPCM (4), i.e., $E\left(x^{*}, \beta\right)=0$, where

$$
E(x, \beta)=G(x)-P_{X}[G(x)-\beta F(x)] .
$$

Lemma 3 [8] Let $x^{*}$ is a solution of $\operatorname{GVI}(F, G, X)(1)$. Then $\forall x \in \mathbb{R}^{n}$

$$
\begin{aligned}
& {\left[G(x)-G\left(x^{*}\right)+\beta\left[F(x)-F\left(x^{*}\right)\right]\right]^{\mathrm{T}} E(x, \beta) \geqslant} \\
& \|E(x, \beta)\|^{2}+\beta\left[F(x)-F\left(x^{*}\right)\right]^{\mathrm{T}}\left[G(x)-G\left(x^{*}\right)\right] .
\end{aligned}
$$

Obviously, it follows from (10) that GPCM (4) is equivalent to the following dynamical system

$$
\frac{\mathrm{d} x}{\mathrm{~d} t}=-E(x, \beta), \quad x\left(t_{0}\right)=x_{0} .
$$

To analyze the exponential stability property of (12), we define a merit function

$V(x)=-\left(x-x^{*}\right)^{\mathrm{T}}\left[\beta F\left(x^{*}\right)+G\left(x^{*}\right)\right]$

$$
+\int_{0}^{1}\left(x-x^{*}\right)^{\mathrm{T}}\left[\beta F\left(x^{*}+\tau\left(x-x^{*}\right)\right)+G\left(x^{*}+\tau\left(x-x^{*}\right)\right)\right] \mathrm{d} \tau,
$$

and its level set

$$
\mathscr{L}\left(x_{0}\right)=\left\{x \in \mathbb{R}^{n} \mid V(x) \leqslant V\left(x_{0}\right)\right\},
$$

where $x^{*}$ is a solution to $\operatorname{GVI}(F, G, X)(1)$, and thus an equilibrium point of (12) due to Lemma 2.

Lemma 4 Let $x^{*}$ is a solution to $\operatorname{GVI}(F, G, X)$ (1). Assume that the following conditions hold:

(a) The function $F$ is G-monotone at $x^{*}$;

(b) The functions $F$ and $G$ are Lipschitz continuous;

(c) The level set $\mathscr{L}\left(x_{0}\right)$ is bounded.

Then, for any initial point $x\left(t_{0}\right)=x_{0} \in \mathbb{R}^{n}$, the trajectory $x(t)$ corresponding to the system (12) converges to this unique solution $x^{*}$, i.e.,

$$
\lim _{t \rightarrow \infty} x(t)=x^{*} .
$$

Proof: Similar to the proof of Theorem 3.4 in [8].

We now begin to establish the stability property of the dynamic system (12).

Theorem 1 Let $x^{*}$ be an equilibrium point of the system (12). Assume that the following conditions hold:

(a) $F(x)$ is a G-strongly monotone function at $x^{*}$;

(b) $G(x)+\beta F(x)$ is a strongly monotone function with modulus $\mu>0$; 
(c) The functions $F$ and $G$ are continuously differentiable;

(d) The matrix $\nabla G(x)+\beta \nabla F(x)$ is symmetric.

Then, the solution trajectory $x(t)$ of (12) is globally exponentially stable at $x^{*}$, i.e., there exist two positive constants $c_{1}$ and $c_{2}$ such that

$$
\left\|x(t)-x^{*}\right\| \leqslant c_{2} e^{-c_{1}\left(t-t_{0}\right)}, \quad \forall t \geqslant t_{0},
$$

where $c_{2}$ is dependent of the initial point $x_{0}$.

Proof: From (13) and the condition (d), it follows that

$$
\nabla V(x)=\beta F(x)+G(x)-\beta F\left(x^{*}\right)-G\left(x^{*}\right) .
$$

Then, by (7), (11), (14), and the condition (a), we have

$$
\begin{aligned}
& \frac{\mathrm{d} V(x(t))}{\mathrm{d} t}=\nabla V(x(t))^{\mathrm{T}} \frac{\mathrm{d} x(t)}{\mathrm{d} t} \\
& \quad=-\left[\beta\left[F(x)-F\left(x^{*}\right)\right]+G(x)-G\left(x^{*}\right)\right]^{\mathrm{T}} E(x, \beta) \\
& \quad \leqslant-\|E(x, \beta)\|^{2}-\beta\left[F(x)-F\left(x^{*}\right)\right]^{\mathrm{T}}\left[G(x)-G\left(x^{*}\right)\right] \\
& \quad \leqslant-\beta\left[F(x)-F\left(x^{*}\right)\right]^{\mathrm{T}}\left[G(x)-G\left(x^{*}\right)\right] \\
& \quad \leqslant-\beta \omega\left\|x-x^{*}\right\|^{2} .
\end{aligned}
$$

This inequality further implies that $V(x(t))$ is monotonically decreasing along the trajectory $x(t)$ in $t$ $\left(\geqslant t_{0}\right)$, and thus

$$
\left\{x(t) \mid t \geqslant t_{0}\right\} \subseteq \mathscr{L}\left(x_{0}\right) .
$$

Using the condition (b), it follows from (14) and Definition 2 that $\nabla V(x)$ is also strongly monotone with modulus $\mu>0$. This fact together with Lemma 1 implies that $V(x)$ is a strongly convex function with modulus $\mu>0$, and hence the level set $\mathscr{L}\left(x_{0}\right)$ ia a bounded and closed convex set.

Since $\mathscr{L}\left(x_{0}\right)$ is a bounded and closed convex set, by (16), the conditions (a) and (c), we deduce from Lemma 4 that

$$
\lim _{t \rightarrow \infty} x(t)=x^{*}, x^{*} \in X^{*} \cap \mathscr{L}\left(x_{0}\right) .
$$

Furthermore, by the boundedness of $\mathscr{L}\left(x_{0}\right)$, it follows from (16) and the condition (c) that the matrix $\nabla G(x)+\beta \nabla F(x)$ is bounded, i.e., there exists a constant $c>0$ such that

$$
\|\nabla G(x(t))+\beta \nabla F(x(t))\| \leqslant c, \quad t \geqslant t_{0} .
$$

Combining (17) with (13) yields

$$
\begin{aligned}
& V(x)=\int_{0}^{1} \tau\left(x-x^{*}\right)^{\mathrm{T}} \times \\
& \quad\left[\int_{0}^{1}\left[\beta \nabla F\left(x_{\theta}\right)+\nabla G\left(x_{\theta}\right)\right]\left(x-x^{*}\right) \mathrm{d} \theta\right] \mathrm{d} \tau \\
& \leqslant \int_{0}^{1} \int_{0}^{1} \tau\left\|\beta \nabla F\left(x_{\theta}\right)+\nabla G\left(x_{\theta}\right)\right\|\left\|x-x^{*}\right\|^{2} \mathrm{~d} \theta \mathrm{d} \tau \\
& \leqslant \int_{0}^{1} \tau\left[\int_{0}^{1} c\left\|x-x^{*}\right\|^{2} \mathrm{~d} \theta\right] \mathrm{d} \tau \\
& =\frac{1}{2} c\left\|x-x^{*}\right\|^{2} .
\end{aligned}
$$

This together with (15) implies that

$$
\frac{\mathrm{d} V(x(t))}{\mathrm{d} t} \leqslant-\beta \omega\left\|x-x^{*}\right\|^{2} \leqslant-\frac{2 \beta \omega}{c} V(x),
$$

and thus

$$
V(x) \leqslant V\left(x_{0}\right) \exp \left[-\lambda\left(t-t_{0}\right)\right], \quad \forall t \geqslant t_{0},
$$

where $\lambda=2 \beta \omega / c$. Note that $V(x)$ is a strongly convex function with modulus $\mu>0$, then

$V(x)-V\left(x^{*}\right) \geqslant \nabla V\left(x^{*}\right)+\mu\left\|x-x^{*}\right\|^{2}=\mu\left\|x-x^{*}\right\|^{2}$.

Combining this inequality with (20) and $V\left(x^{*}\right)=0$ yields

$$
\left\|x-x^{*}\right\| \leqslant \sqrt{\frac{V(x)}{\mu}} \leqslant \sqrt{\frac{V\left(x_{0}\right)}{\mu}} \mathrm{e}^{\left(-\frac{\lambda\left(t-t_{0}\right)}{2}\right)}, \quad \forall t \geqslant t_{0},
$$

and the desired assertion follows with $c_{1}=\lambda / 2$ and $c_{2}=\sqrt{V\left(x_{0}\right) / \mu}$. This proof is completed.

Remark 1 From Theorem 1, we see that the stability property of GPCM (4) is established without requiring an upper bound of the matrix $\nabla G(x(t))+$ $\beta \nabla F(x(t))$, while the condition is assumed to prove the stability property of GNPP (2) in [7]. Therefore, the system GPCM (4) can be applied to analyze a much wider range of dynamical system, and thus it is more practical.

Obviously, for any $\beta>0$, it follows from Definition 2 that the function $\beta F(x)+x$ (or $\beta x+G(x)$ ) is strongly monotone if $F(x)$ (or $G(x)$ ) is monotone. Thus, we obtain the following stability results, which are immediate corollaries of Theorem 1.

Corollary 1 Let $G(x)=x$. Assume that the function $F$ is continuously differentiable and monotone. If the matrix $\nabla F(x)$ is symmetric, then the dynamic system (12) with any initial point $x_{0} \in X$ converges to the unique solution $x^{*}$ of $\operatorname{GVI}(F, G, X)$ (1) exponentially. 
Corollary 2 Let $F(x)=x$. Assume that the function $G$ is continuously differentiable and monotone. If the matrix $\nabla G(x)$ is symmetric, then the dynamic system (12) with any initial point $x_{0} \in X$ converges to the unique solution $x^{*}$ of $G V I(F, G, X)$ (1) exponentially.

Remark 2 Compared with the conclusions of Corollary 1 (or Corollary 2) in [7], the exponential stability of GPCM (4) in Corollary 1 (or Corollary 2) here is established without requiring the strong monotonicity of $F$ (or $G$ ) and an upper bound of the matrix $\nabla F(x)$ (or $\nabla G(x)$ ), but the monotonicity of $F$ (or $G$ ). Therefore, our conclusions are more practical.

Acknowledgements: This work is Supported by NNSF of China (Nos. 11961018, 61762032) and NSF of Hainan Province (No. 2016CXTD004.

\section{REFERENCES}

1. Noor MA (1998) General nonlinear variational inequalities. Appl Math Lett 1, 119-121.
2. Harker PT, Pang JS (1990) Finite dimensional variational inequality and nonlinear complementarity problems: A survey of theory, algorithms and apllications. Math Program 48, 161-220.

3. Giannessi F, Maugeri A (1995) Variational Inequalities and Network Equilibrium Problems, Plenum Press, NY.

4. Li M, Liao LZ, Yuan XM (2009) Proximal point algorithms for general variational inequalities. J Optim Theory Appl 142, 125-145.

5. He BS (1999) Inexact implicit methods for monotone general variational inequalities. Math Program 86, 199-217.

6. Liao LZ, Qi HD, Qi LQ (2004) Neurodynamical optimization. J Global Optim 28, 175-195.

7. Xia YS, Wang J (2004) A general projection neural network for solving monotone variational inequalities and related optimization problems. IEEE Trans Neural Netw 15, 318-328.

8. Ou YG, Lin HC (2016) A continuous method model for solving general variational inequality. Int $J$ Comput Math 93, 1899-1920.

9. Sun WY, Yuan YX (2006) Optimization Theory and Methods: Nonlinear Programming, Springer, NY. 